\title{
Semi-quantitative analysis on the content of berberine hydrochloride in compound berberine tablets with the fluorescence spectral imaging method
}

\author{
Lan Liang*, ${ }^{*}$ Jinyan Sun*, Qing $\mathrm{He}^{*, \dagger}, *$, Zhenqiang Chen ${ }^{\dagger}$, \\ Siqi $\mathrm{Zhu}^{\dagger}$ and Lin Lin* \\ *Department of Biomedical Engineering \\ Guangdong Medical University \\ Dongguan 523808, P. R. China \\ ${ }^{\dagger}$ Department of Optoelectronic Engineering \\ Jinan University, Guangzhou 510630, P. R. China \\ *jessiehq@163.com
}

Received 1 July 2015

Accepted 13 November 2015

Published 24 December 2015

\begin{abstract}
The content of berberine hydrochloride (BH) in compound berberine tablets (CBTs) is subject to strict requirements. Its content is usually measured based on chemical analysis. In this paper, the fluorescence spectral imaging method was used to study the relative content of $\mathrm{BH}$ from a physics perspective. By comparing the relative fluorescence intensity of self-made CBTs with different mass percentages of $\mathrm{BH}$, a linear positive relationship was observed between the $\mathrm{BH}$ content and the relative fluorescence intensity, and accordingly the quality of CBTs of different brands was evaluated. The results indicate that the fluorescence spectral imaging method can be a simple, fast and nondestructive semi-quantitative analysis method to determine the content of $\mathrm{BH}$ in CBTs, and this method has great potential in the quality control of CBTs.
\end{abstract}

Keywords: Fluorescence spectral imaging; compound berberine tablet; berberine hydrochloride; semi-quantitative analysis.

\section{Introduction}

The compound berberine tablet (CBT) is a kind of Chinese patent medicine (CPM). It is composed of berberine hydrochloride $(\mathrm{BH})$, radix aucklandiae, fructus evodiae, radix paeoniae alba. Its effects include cleaning heat, drying humidity, promoting qi circulation, relieving pain and curing durchfall. It is mostly used to treat diseases like damp-heat in the large bowel, diarrhea and tenesmus. $\mathrm{BH}$ is the antibacterial ingredient in CBT, and the daily recommended dosage is less than $0.9 \mathrm{~g}$. According to the Chinese pharmacopoei (2010), CBT (0.5 g)

\$Corresponding author.

This is an Open Access article published by World Scientific Publishing Company. It is distributed under the terms of the Creative Commons Attribution 4.0 (CC-BY) License. Further distribution of this work is permitted, provided the original work is properly cited. 


\section{Liang et al.}

should have $30 \mathrm{mg}$ of $\mathrm{BH}$, and its content should be tested by high performance liquid chromatography (HPLC). ${ }^{1}$ Because of this regulation, whether the $\mathrm{BH}$ content meets the criterion is an important indicator to evaluate the quality of the CBT. Besides the HPLC, the BH content can also be determined by ultraviolet spectrophotometry. ${ }^{2}$ However, these methods require chemical preprocessing of the sample, ${ }^{2,3}$ which can cause damage to the sample.

In recent years, the spectral imaging method has been widely used in biomedical, food quality evaluation and other areas, and it has obtained significant results. ${ }^{4,7}$ Spectral imaging technology refers to the use of multiple spectral channels for image acquisition, display, processing, analysis and interpretation. It uses area array CCD devices and various optical imaging methods (e.g., telescopic, photographic and microscopic systems) to obtain images of the sample, and the point in the twodimensional image corresponds to one point in the sample. The optical parameters of the image points, such as light intensity and gray value, directly reflect the optical parameters of the corresponding points in the sample. Thus, the physical and chemical characteristics (reflectivity, scattering rate, component content, etc.) at each point of the sample can be calculated based on the optical image. Today, spectral imaging technology can automatically change the work wavelength to obtain continuous photographs of the sample under different wavelengths, thus obtain the distribution image of the physical and chemical properties of the sample. These images show the optical spectrum curves of each corresponding point. Finally, from the peak-to-valley position and intensity change of the spectrum curve, qualitative and quantitative analytics information can be obtained. ${ }^{8,9}$ Also, fluorescence images of the sample excited by short waves are gathered by the fluorescence spectral imaging method. Fluorescence phenomena are connected with the molecular structure and component content of the material, ${ }^{10}$ so that the fluorescence spectrum can be used to identify material and carry out quantitative analysis.

The fluorescence spectral imaging method can be easily used, and it does not destroy the molecular structure of the sample during the detection process. Thus, it has important application significance in the field of traditional Chinese medicine. ${ }^{11-13} \mathrm{In}$ this study, the fluorescence spectral imaging method is used to conduct semi-quantitative analysis on the content of $\mathrm{BH}$ in $\mathrm{CBT}$ in an attempt to establish a new method to determine the ingredient content of CPM and provide a new way to evaluate the quality of CBT.

\section{Methods}

\subsection{Fluorescence spectral imager}

The fluorescence spectral imager ${ }^{14}$ (Fig. 1) used in this study consists of a UV light source with a center wavelength of $254 \mathrm{~nm}$, a VariSpec liquid crystal tunable filter (LCTF), a receiving unit including a lens and an area array CCD, an image acquisition card and a computer. The working wavelength range of LCTF was from $400 \mathrm{~nm}$ to $720 \mathrm{~nm}$, and the scanning step was set to $5 \mathrm{~nm}$. The exposure time of CCD was $800 \mathrm{~ms}$. The receiving unit worked in a continuous mode, and synchronized with the spectral scanning.

The sample was placed on the substrate during testing, and emitted fluorescence after being excited by UV light. The fluorescence was divided into several narrow spectral bands in the time dimension after passing through the LCTF. It was then imaged on the CCD target surface and stored in the computer using the image acquisition card. Each testing sample produced a spectral image sequence that contained 65 images (one image every $5 \mathrm{~nm}$ from $400 \mathrm{~nm}$ to $720 \mathrm{~nm}$ ). After eliminating the image noise with a band-pass filter, the active pixels were determined for each image. The relative fluorescence intensity at each wavelength was calculated by averaging the gray value of all of the active

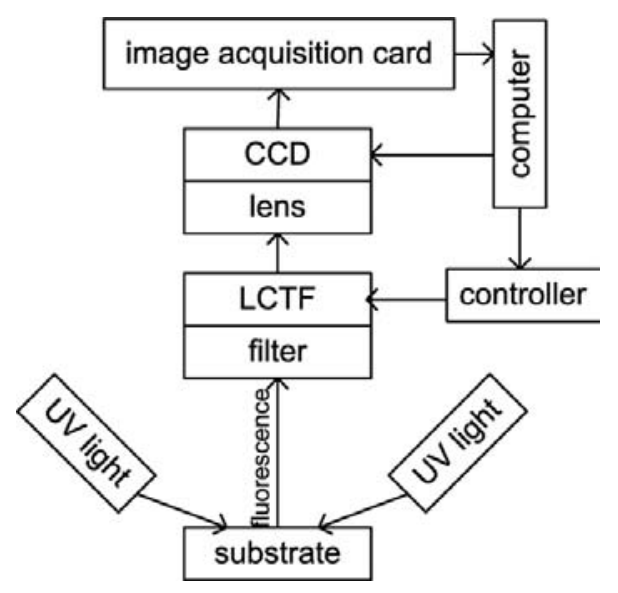

Fig. 1. Spectral imaging system block diagram. 


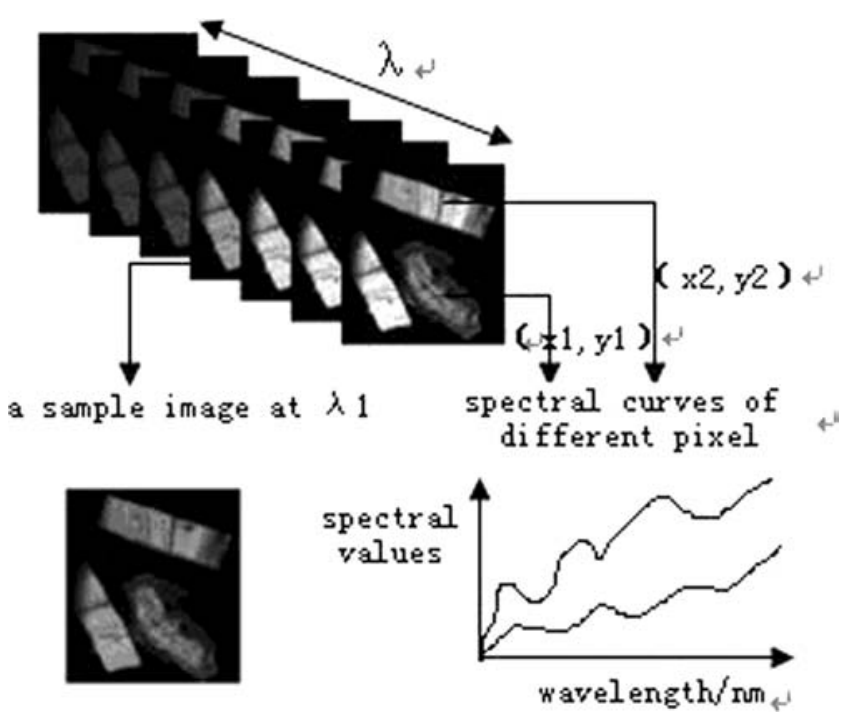

Fig. 2. Hyperspectral image block of a sample.

pixels for each image. Finally, the sample's fluorescence spectrum curve was obtained (Fig. 2).

\subsection{Sample preparation}

In this study, the radix paeoniae alba, radix aucklandiae, fructus evodiae and $\mathrm{BH}$ used to produce the self-made CBT were bought from a medicinal materials market. Four negative CBTs were made: NPC1 (without radix aucklandiae), NPC2 (without fructus evodiae), NPC3 (without radix paeoniae alba) and NPC4 (without $\mathrm{BH}$ ). The standard $\mathrm{BH}$ (batch number: 110713-200609), the radices paeoniae alba reference material (batch number: 120905201109), the radix aucklandiae reference material (batch number: 120921-201008) and the fructus evodiae reference material (batch number: 120909200508) were supplied by National Institutes for Food and Drug Control.

A brand of CBT was chosen as the subject for semi-quantitative analysis. The quality of the CBT was about $0.445 \mathrm{~g}$ weighed with electronic balance, so the mass percentage of $\mathrm{BH}$ in each $\mathrm{CBT}$ was $6.74 \%$ according to the requirement of Chinese pharmacopeia. We could calculate the content of $\mathrm{BH}$ in the CBT sample of different weight based on the percentage. Simple processing was carried out on the samples, and the resulting details are shown in Table 1.

In order to further investigate the relationship between fluorescence intensity change and the content of $\mathrm{BH}$, the $\mathrm{CBT}$ was also self-made
Table 1. A brand of CBT with different mass percentages of $\mathrm{BH}$ used in semi-quantitative analysis.

\begin{tabular}{|c|c|c|c|c|c|}
\hline Total weight (g) & $2 \mathrm{~g}$ & $2 \mathrm{~g}$ & $2 \mathrm{~g}$ & $2 \mathrm{~g}$ & $2 \mathrm{~g}$ \\
\hline $\begin{array}{l}\text { CBT sample weight } \\
\text { (g) }\end{array}$ & 1.85 & 1.80 & 1.75 & 1.70 & 1.65 \\
\hline $\begin{array}{l}\text { BH content in the } \\
\text { sample }(\mathrm{g})\end{array}$ & 0.125 & 0.121 & 0.118 & 0.114 & 0.111 \\
\hline $\begin{array}{l}\text { BH content added to } \\
\text { sample }(\mathrm{g})\end{array}$ & 0.15 & 0.20 & 0.25 & 0.30 & 0.35 \\
\hline Total BH content $(\mathrm{g})$ & 0.275 & 0.321 & 0.368 & 0.414 & 0.461 \\
\hline $\begin{array}{l}\text { Mass percent of } \\
\text { BH }(\%)\end{array}$ & 13.75 & 16.05 & 18.4 & 20.7 & 23.05 \\
\hline
\end{tabular}

according to Chinese pharmacopoei's prescription (2010). $2.32 \mathrm{~g}$ of radix aucklandiae, $0.8 \mathrm{~g}$ of fructus evodiae and $3.24 \mathrm{~g}$ of radix paeoniae alba were mixed with $\mathrm{BH}$ of different weights $(0.24,0.60,1.00$, 1.46 and $1.97 \mathrm{~g}$ ), forming the CBTs with different mass percentages of $\mathrm{BH}(3.62 \%, 8.62 \%, 13.62 \%$, $18.62 \%$ and $23.62 \%$ ).

11 types of CPM from different manufacturers with $\mathrm{BH}$ as the major ingredient were selected (Table 2). Among these 11 types of CPM, five are called $\mathrm{BH}$ tablets ( $\mathrm{S} 1-\mathrm{S} 5$ ), one is named as $\mathrm{BH}$ capsule ( $\mathrm{S} 6)$, one is called a compound ancklandia and berberine tablet (S7) and four are called CBT (S8-S11).

Table 2. 11 types of CPM samples from different manufacturers.

\begin{tabular}{llccc}
\hline Number & \multicolumn{1}{c}{ Name } & $\begin{array}{c}\text { Main } \\
\text { ingredients }\end{array}$ & $\begin{array}{c}\text { Tablet/ } \\
\text { capsule } \\
\text { weight }(\mathrm{g})\end{array}$ & $\begin{array}{c}\text { BH } \\
\text { weight } \\
(\mathrm{g})\end{array}$ \\
\hline S1 & BH tablet & BH & 0.1 & 0.1 \\
S2 & BH tablet & BH & 0.1 & 0.1 \\
S3 & BH tablet & BH & 0.1 & 0.1 \\
S4 & BH tablet & BH & 0.1 & 0.1 \\
S5 & BH tablet & BH & 0.1 & 0.1 \\
S6 & BH capsule & BH & 0.1 & 0.1 \\
S7 & compound & BH, RA, FE & 0.48 & 0.05 \\
& ancklandia and & & & \\
S8 & berberine tablet & BH, RA, & 0.45 & 0.03 \\
& CBT & FE, RPA & & \\
S9 & CBT & BH, RA, & 0.45 & 0.03 \\
S10 & CBT & FE, RPA & & \\
& & BH, RA, & 0.45 & 0.03 \\
S11 & CBT & FE, RPA & & \\
& & BH, RA, & 0.45 & 0.03 \\
& & FE, RPA & & \\
\hline
\end{tabular}

RA, FE, RPA are short for radix aucklandiae, fructus evodiae and radix paeoniae alba, respectively. 


\section{Liang et al.}

To better observe the fluorescence of the samples, all the samples were preprocessed before the test. For each CPM, two capsules/tablets were selected, ground into powder, filtered though a mesh sieve and pressed into tablets. The self-made negative product control (NPC) and the self-made CBT of various $\mathrm{BH}$ contents were also preprocessed in the same way.

\section{Results and Discussion}

\subsection{The fluorescence comparison between medicinal materials and their reference materials}

Figure 3 shows the fluorescence spectrum curves for radix paeoniae alba, radix aucklandiae, fructus evodiae, $\mathrm{BH}$ and their standard reference materials. The curve shape and peak-to-valley position of the spectrum curves showed that the fluorescence spectrum curves for radix paeoniae alba, radix aucklandiae, fructus evodiae and $\mathrm{BH}$ were very similar to their standard reference materials, suggesting that the high quality of these four Chinese medicinal materials has guaranteed the quality of the self-made CBT. ${ }^{14,15}$

\subsection{The fluorescence comparison between NPCs}

The fluorescence spectrum curves for the four NPCs are shown in Fig. 4. From $520 \mathrm{~nm}$ to $670 \mathrm{~nm}$, the

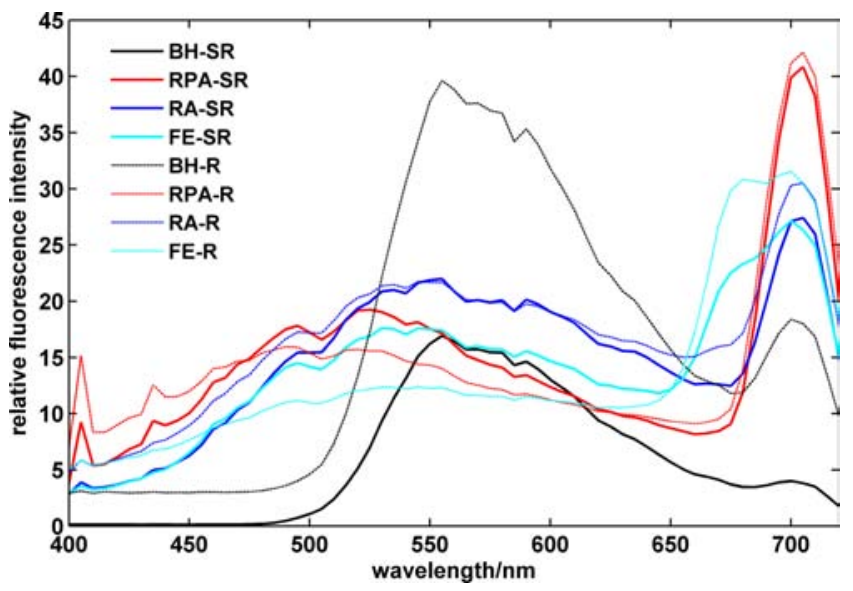

Fig. 3. The relative fluorescence for radix paeoniae alba (RPA-R), radix aucklandiae (RA-R), fructus evodiae (FE-R), $\mathrm{BH}(\mathrm{BH}-\mathrm{R})$ and their corresponding standard reference materials (RPA-SR, RA-SR, FE-SR and BH-SR).

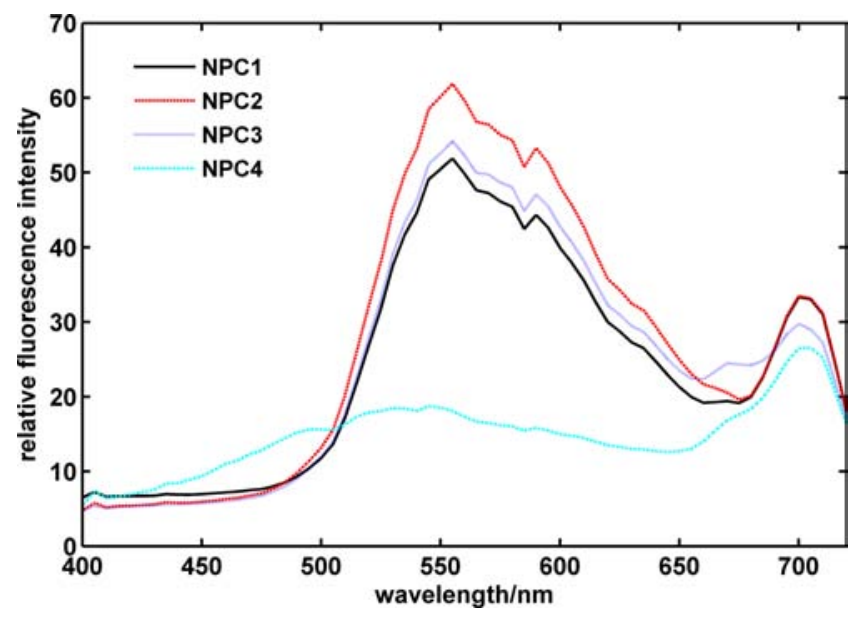

Fig. 4. The relative fluorescence intensity for the four negative CBTs.

relative fluorescence for $\mathrm{NPC} 1 / \mathrm{NPC} 2 / \mathrm{NPC} 3$ was stronger than that of NPC4, which indicates that the $\mathrm{BH}$ contributed the most to the fluorescence intensity of the CBT in this band range. This result indicates that relative fluorescence intensity can be used to semi-quantitatively analyze the $\mathrm{BH}$ content in CBT. Meanwhile, the shape of the fluorescence spectrum curve of NPC4 was evidently different from the other three, and this information can be used in quality control of CBT from the market. ${ }^{16}$

\subsection{The semi-quantitative analysis of $\mathrm{BH}$}

For different mass percentages of CBT, Fig. 5 shows that their fluorescence intensity did not increase gradually with the increase of the mass percentage.

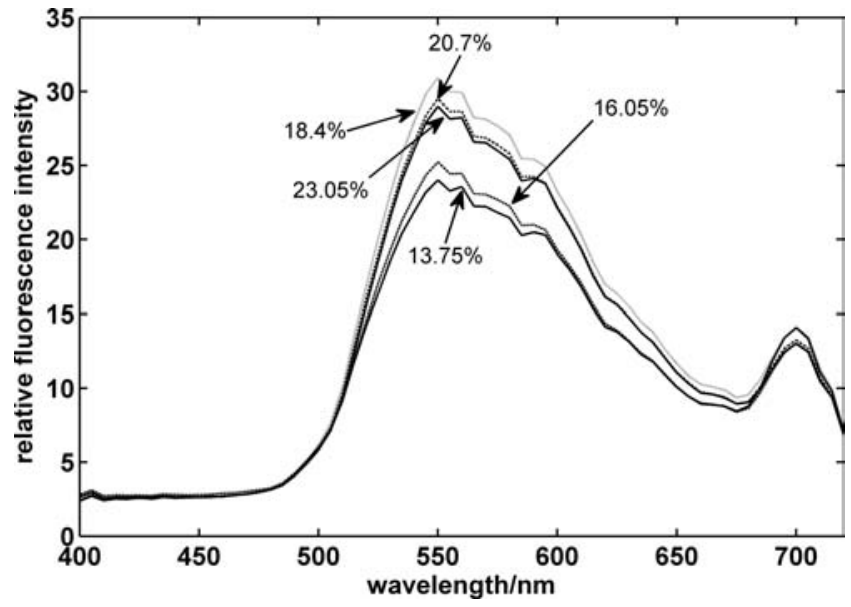

Fig. 5. The comparison of relative fluorescence intensity between CBT with different mass percentages of $\mathrm{BH}$. 


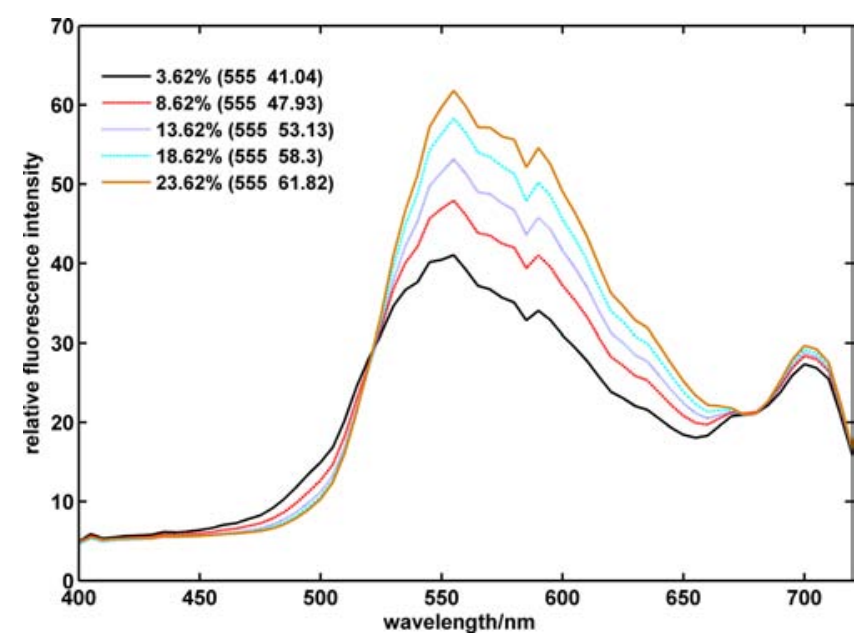

Fig. 6. The relative fluorescence intensity for the self-made CBT with different mass percentages of $\mathrm{BH}$. The numbers in the brackets show that the maximal fluorescence appears at $555 \mathrm{~nm}$.

For the first three samples, the fluorescence intensity increased as the $\mathrm{BH}$ was increased, but the relationship was not linear. The reason may be that the percentage of $\mathrm{BH}$ content in samples did not meet the provision. Conversely, the fluorescence intensity of the last two samples was lower than the $18.4 \%$ of CBT. Besides the influence of $\mathrm{BH}$ in the sample itself, one reason may be that the added quality of $\mathrm{BH}$ was too high, which made the concentration of $\mathrm{BH}$ in samples increase, thus leading to fluorescence self-quenching, causing the intensity to not enhance. ${ }^{10,17-19}$

Moreover, the relationship between the mass percentage of $\mathrm{BH}$ in self-made $\mathrm{CBT}$ and its fluorescence intensity was evaluated. Figure 6 shows that the content increase of $\mathrm{BH}$ led to an enhancement of the fluorescence excitation. The linear regression based on the least square method was applied, and the results indicated that the mass percentage of $\mathrm{BH}(x)$ was significantly linearly correlated with the fluorescence maximum $\left(y_{1}\right)$ $\left(y_{1}=11.87 x+39.93, R^{2}=0.987, p<0.001\right)$ and the mean fluorescence intensity $\left(y_{2}\right)$ from $520 \mathrm{~nm}$ to $670 \mathrm{~nm}\left(y_{2}=7.21 x+28.8, R^{2}=0.999, p=0.003\right)$. This shows that the relative fluorescence intensity of a sample of the self-made CBT can predict its $\mathrm{BH}$ content. ${ }^{20,21}$

\subsection{The quality evaluation of CBT}

Based on the results of the semi-quantitative analysis, fluorescence measurement was conducted on



Fig. 7. The relative fluorescence intensity for the standard BH (S0) and the CPM samples (S1-S11).



(a)

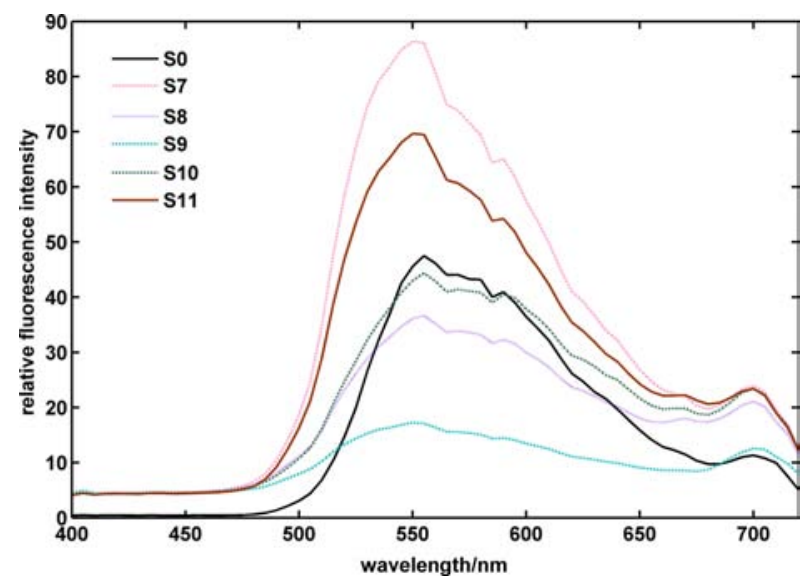

(b)

Fig. 8. (a) The fluorescence comparison between different kinds of $\mathrm{BH}$ tablets/capsules; (b) the fluorescence comparison between different kinds of CBTs. 


\section{Liang et al.}

the 11 types of CPM samples (Table 1 ). Figure 7 shows the fluorescence spectrum curves for each $\mathrm{CPM}$ and the standard $\mathrm{BH}$. The shapes of the fluorescence curves for most of the CPM samples have a great similarity to the standard BH. Sample 2 has a distinct pattern in which its fluorescence remained almost constant between $550 \mathrm{~nm}$ and $600 \mathrm{~nm}$. This indicated that the quality of sample 2 was significantly different from the other 10 samples that were made by different manufacturers. For S1S6, two tablets or capsules were used for testing, totaling $0.2 \mathrm{~g}$ of $\mathrm{BH}$. Therefore, it was expected that the relative fluorescence for each type of $\mathrm{BH}$ tablet would be almost the same. In fact, however, the maximum of the relative fluorescence intensity for these six kinds of $\mathrm{BH}$ tablets/capsules varied significantly: from 65.08 (S2) to 175.7 (S3) (Fig. 8(a). It was the same with the CBTs (Fig. 8(b). Besides, the relative fluorescence for $\mathrm{S} 7$ and $\mathrm{S} 11$ was even stronger than some of the $\mathrm{BH}$ tablet samples $(0.2 \mathrm{~g}$ of $\mathrm{BH})$. These results reveal the different contents of $\mathrm{BH}$ for each sample, and the unstable quality of the CBTs, ${ }^{22,23}$ and suggest that this spectral imaging method can be used for quality evaluation of CPM and Chinese medicinal materials. ${ }^{24}$

\section{Conclusion}

In this study, the content of the $\mathrm{BH}$ in the self-made CBT was semi-quantified by the fluorescence spectral imaging method. The results show that there was a positive linear relationship between the mass percentage of the $\mathrm{BH}$ in self-made $\mathrm{CBT}$ and the relative fluorescence intensity; higher mass percentages induced stronger fluorescence intensity. This linear relationship can be used for semi-quantitative analysis of the BH content in CPM. Additionally, the fluorescence measurements of the 11 types of CBTs suggest that their BH contents are significantly different. This quality instability further emphasized that quality control of CPM should be enhanced.

Further investigation of the linear relationship between the $\mathrm{BH}$ mass percentage and the fluorescence intensity should be conducted for a wider range of $\mathrm{BH}$ mass percentages. Also, uniting HPLC with the correlation mathematical modeling method, we want to establish a direct relation between the fluorescence intensity and the content of $\mathrm{BH}$, which helps to indicate whether the $\mathrm{BH}$ content meets the criteria of the Chinese pharmacopoei.

\section{Acknowledgment}

The authors would like to acknowledge the support of the Ph.D. research startup foundation of Guangdong Medical University (2XB14006).

\section{References}

1. N. P. Committee, "Pharmacopoeia of the People's Republic of China, 2010 Edition, Volume I," 392-393 (2010).

2. R. Wang, M. He, X. Yuan, Y. Zhou, M. Xue, "Study on the method of TLC UV determine the content of berberine hydrochloride in compound berberine tablets," Guangdong Chem. Ind. 39(1), 112, 104 (2012)

3. X. He, C. Zuo, "Determination of berberine in compound berberine tablets by HPLC," West China J. Pharm. Sci. 14(2), 132-133 (1999).

4. A. Machikhin, V. Pozharand, V. Batshev, "DoubleAOTF-based aberration-free spectral imaging endoscopic system for biomedical applications [J]," J. Innov. Opt. Health Sci. 08(03), 1541009 (2015).

5. R. Zhang, L. Yin and S. Jin, "Detection of illegally added drugs in dietary supplements by near-infrared spectral imaging $[\mathrm{J}]$, , J. Innov. Opt. Health Sci. $\mathbf{0 7}$ (06), 1450032 (2014).

6. D. Wang, Y. Ding, Z. Guo, S. Min, "The application of near-infrared spectra micro-image in the imaging analysis of biology samples $[\mathrm{J}]$, , J. Innov. Opt. Health Sci. 07(04), 1350062 (2014).

7. Z. Yang, C. Wang, L. Han, J. Li, X. Liu. "Rapid screening and visual tracing of melamine in soybean meal by NIR microscopy imaging $[\mathrm{J}], " J$. Innov. Opt. Health Sci. 07(04), 1350072 (2014).

8. S. Fan, Y. Li, Y. Zhao, "Principle, techique, and biomedical applications of spectral imaging $[\mathrm{J}]$," Life Sci. Instrum. 4, 003 (2004).

9. F. Shifu, X. Songshan, Z. Youquan, L. Yun, Z. Yuchun, "Spectral imaging technique and its biomedical researching applications," Mod. Sci. Instrum. 1, 020 (2005).

10. J. R. Lakowicz, Principles of Fluorescence Spectroscopy (Springer Science \& Business Media, 2013).

11. Q. He, L. Liang, J. Zhao, J. Ma, "Research on application of fluorescence spectrum imaging method in banlangen granule quality evaluation," Spectrosc. Spect. Anal. 33(11), 3044-3049 (2013).

12. L. Liang, L. Wang, J. Zhao, J. Ma, "Study of cortex phellodendri chinensis decoction experiment based on the spectral imaging technology," Spectrosc. Spect. Anal. 32(5), 1359-1361 (2012).

13. I. Vermaak, A. Viljoen, S. W. Lindström, "Hyperspectral imaging in the quality control of herbal medicines - The case of neurotoxic Japanese 
star anise," J. Pharm. Biomed. Anal. 75, 207-213 (2013).

14. J. Zhao, "Design of a continuous spectrum imaging apparatus based on LCTFs," Acta Photonica Sin. 37(4), 758 (2008).

15. J. Zhao, Q. Pang, J. Ma, C. Liu, L. Wang, D. Cui, "In vivo identification of radix panacis quinquefolii by spectral imaging technology," Spectrosc. Spect. Anal. 31(1), 210-213 (2011).

16. G. Xi, S. Chen, "Content determination of berberine hydrochloride in compound berberine tablets," World Chin. Med. 10(2), 252-254 (2015).

17. H. Li, Y. Chen, C. Yan, Y. Hu, "Fluorescence self quenching and the quenching mechanism of magnolol," Acta Phys.-Chim. Sin. 23(9), 1454-1458 (2007).

18. J. Zhang, J. Wang, S. Wang, X. Lu, "Fluorescence spectra of 5-sulfosalicylic acid and self quenching," Energy Res. Manage. (4), 54-56 (2011).

19. N. He, F. Zhong, Y. Shu, Y. Sun, H. Sui, $\mathrm{X}$. Hao, "Synthesis and fluorescence properties of phenylquinoline/acrylates copolyme," J. Funct. Mater. 43(16), 2156-2160 (2012).

20. Y. Shen, B. Xu, "Determination of concentration of berberine hydrochloride in various drugs of compatibility of coptis chinensis," J. Harbin Univ. of Commer. 31(2), 155-158 (2015).

21. J. Yuan, K. Wang, "Determination of berberine hydrochloride in Wei'an tablets by RP-HPLC," Anhui Med. Pharm. J. 19(6), 1084-1085 (2015).

22. D. Guo, Y. Lin, J. Liu, L. Lin and B. Wang, "Research on dissolution profiles of compound Huangliansu tablets from different manufacturers," J. Pharm. Res. 32(4), 208-210 (2013).

23. L. Lin, Y. Lin, B. Wang, D. Guo, Y. Zang, L. Xu, "Quality analysis of Fufang Huangliansu tablet," J. Pharm. Res. 32(3), 157-158 (2013).

24. F.-B. Lin, Q.-C. Pang, J. Ma, J. Zhao, Z.-L. Li, W.-X. Zhang, "Identification of herbal medicines based on spectral imaging detection," J. Appl. Opt. 2, 025 (2010). 